\title{
Bricks and 17 Clicks
}

Nikhil Panda, MD

I can remember texting my medical school friends, "Good luck!" at 9:30 PM the night before the first day of surgical internship. Although simply intended to be an opportunity to connect with my classmates one last time before starting on the 7-year journey ahead, those 8 letters resulted in a 50-plus message thread continuing until midnight. Each green bubble of text reminded me that I wasn't the only one who was simultaneously too excited and anxious to put myself to bed. None of us knew that in a few short weeks, we would all have developed the ability to sleep on command, something that would infuriate our partners on rare date nights.

In those messages, each of us respected the challenges ahead, but stood tall with pride in our preparedness. After all, we had just graduated from a medical school with an especially rigorous surgical clerkship and subinternship. Work hours for medical students were less regulated. Burnout didn't apply to us-or so we thought.

On the day I realized I was wrong, I was halfway through my second year of residency on an on-call weekend. It was a tough day by anyone's standards and a tough time of year for me. The line between the hospital and everywhere else was blurred by the winter cold and the sun setting before I left the wards each day. But, no worries-there were 2 appendectomies, in young and thin patients, scheduled for that evening, and I was determined to staple, bag, and extract both appendixes. All that was standing between the operating room and me was sign-out from the day teams, a quick walk through the intensive care unit (ICU), and, if I was lucky, a stop at the nurses' station for a dinner of saltines and peanut butter.

Sign-out began. The lists were long, and the patients were sick. With each patient, I saw a brick fall to the ground, building a wall between the operating room and me. My pager rang and interrupted sign-out with, "Patient requesting stool softener," and "Family at bedside, requesting to speak with surgeon." Two more bricks. My colleague was nearing the end of the list when she arrived at the last

DOI: http://dx.doi.org/10.4300/JGME-D-19-00034.1 patient, who, for purposes of anonymity, we will call Susan.

Susan was an elderly woman who had undergone emergency surgery weeks earlier for a perforated viscus. While she survived surgery, she had remained intubated and critically ill in the ICU with septic shock. My colleague began to tell me that there had been a family meeting earlier in the day to discuss Susan's goals of care. My pager rang: "Could you please discontinue telemetry for room 74-A?" Brick 3. Back to Susan. At the meeting, Susan's family had decided to withdraw supportive care and focus on comfort measures for their loved one. My colleague and I exchanged a few words about how unfortunate this situation must be for Susan and her family, as we knew she was likely to pass away soon.

"Is the death paperwork prepared?" I asked, thinking about the brick wall I had to climb to even glimpse the operating room. I don't remember my colleague's answer because, in that moment, I felt a sudden mixture of shame and disappointed surprise. This is the moment when a person's entire life, and its meaning to family, friends, and physicians, became just another brick in my selfish and imaginary wall.

Burnout, in my mind, was always attached to something physical: losing or gaining 15 pounds during internship, yelling and slamming a door, crying at the end of a long day, or sleeping too little or too much. ${ }^{1}$ In response to the high prevalence of burnout among surgical and other trainees, ${ }^{2}$ there have been many changes to work hour standards. Training programs are now working to prioritize resident well-being, remove the stigma to seeking support, ${ }^{3}$ and promoting a healthy work-life balance among all providers. My training program even converted call rooms into a small gym near the entrance to our surgical wards. Despite these changes in culture, my idea of burnout remained linked to a scene on replay of climbing the steepest mountain only to fall backward when nearing the apex, out of energy and without a soft landing. None of this had resonated with me as of yet during my surgical training. Until Susan, I thought I was above burnout.

Sharing this experience with my colleagues (my classmates and I still have the same group messaging thread) and mentors returned a surprising number of similar experiences. It turns out that for some, burnout is the moment when we lose focus of our 
truest motivations, a dimension of burnout known as depersonalization. ${ }^{4}$ It's when a young man with an inflamed appendix becomes "appendicitis," and we forget his pain during the physical examination, but remember his habitus because it may make the surgery more straightforward. It's when an appendectomy becomes "staple, bag, and extract," rather than a careful operation to alleviate symptoms or prevent a devastating complication.

To this day, I feel ashamed of how I acted during sign-out on Susan. I went back into our electronic health record, and I counted the number of clicks with a mouse it takes to complete the death paperwork for a patient. I counted 17 clicks. For those of you who text from a smartphone, that's fewer than the number of screen taps it takes to open up your messages and shoot a quick, "Running late, sorry!" (22 clicks) or "Can't wait to see you tonight, long day..." (40 clicks). The bricks that fell in front of me didn't represent a hurdle to making it into an operating room that night. Each was a manifestation of my own personal burnout; the last brick fell the hardest, when I realized I equated a person's life to 17 clicks with a mouse.

I never made it into the operating room that night to assist in any surgeries. Instead, I was in the ICU with Susan and her family and was the physician who pronounced her passing standing next to 3 generations of her loved ones.

\section{References}

1. Dzau VJ, Kirch DG, Nasca TJ. To care is humancollectively confronting the clinician-burnout crisis. $N$ Engl J Med. 2018;378(4):312-314. doi:10.1056/ NEJMp1715127.

2. Lebares CC, Guvva EV, Ascher NL, O'Sullivan PS, Harris HW, Epel ES. Burnout and stress among US surgery residents: psychological distress and resilience. J Am Coll Surg. 2018;226(1):80-90. doi:10.1016/j. jamcollsurg.2017.10.010.

3. Gerada C, Jones R. Surgeons and mental illness: a hidden problem? BMJ. 2014;348:g2764. doi: https://doi.org/10. 1136/bmj.g2764.

4. Thomas NK. Resident burnout. JAMA. 2004;292(23):2880-2889. doi:10.1001/jama.292.23. 2880 .

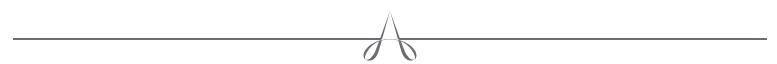

Nikhil Panda, MD, is a General Surgery Resident, Department of Surgery, Massachusetts General Hospital.

Corresponding author: Nikhil Panda, MD, Massachusetts General Hospital, Department of Surgery, GRB-425, 55 Fruit Street, Boston, MA 02114, 617.726.2800, fax 617.724.3499,

nikhil.panda@mgh.harvard.edu 